\title{
The role of CT in PET/CT for assessing diffuse infiltration of bone marrow in multiple myeloma using the Durie-Salmon PLUS staging system
}

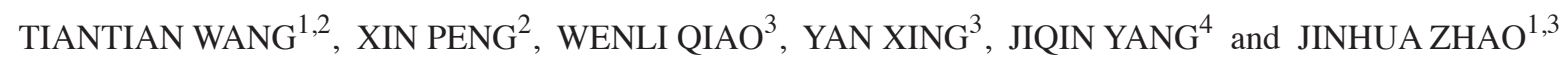 \\ ${ }^{1}$ Department of Nuclear Medicine, Shanghai General Hospital of Nanjing Medical University, Shanghai 200080; \\ ${ }^{2}$ Department of Radiology, Tengzhou Central People's Hospital, Tengzhou, Shandong 277500; ${ }^{3}$ Department of \\ Nuclear Medicine, Shanghai General Hospital, Shanghai Jiao Tong University School of Medicine, Shanghai 200080; \\ ${ }^{4}$ Department of Nuclear Medicine, General Hospital of Ningxia Medical University, Yinchuan, Ningxia 750004, P.R. China
}

Received January 23, 2019; Accepted March 16, 2020

DOI: $10.3892 / \mathrm{mco} .2020 .2039$

\begin{abstract}
PET/CT has been identified as one of the routine methods for the assessment of multiple myeloma (MM) bone marrow infiltration. In the routine method of performing $\mathrm{PET} / \mathrm{CT}$, the $18 \mathrm{~F}-\mathrm{Fludeoxyglucose}\left({ }^{18} \mathrm{~F}-\mathrm{FDG}\right)$ uptake in this disease is often used in the assessment of this condition, however CT diagnosis is not currently commonly used. The aim of the present study was to investigate the importance of $\mathrm{CT}$ in PET/CT for assessing diffuse infiltration (DI) of bone marrow in MM. MRI was used as a control in the present study, which is the gold standard for assessing DI of bone marrow and is divided into 3 levels: Mild, moderate and severe DI. Subsequently, a total of four combinations of PET and CT results were listed using the enumeration method for the evaluation of DI in the bone marrow. These combinations were respectively compared with the three levels of MR imaging to screen the most consistent method. The concordances of the new method and routine ${ }^{18} \mathrm{~F}-\mathrm{FDG} \mathrm{PET} / \mathrm{CT}$ for the assessment of DI with MR imaging were compared using the McNemar test, respectively. The results of the DI assessment from the two methods were verified by performing Durie-Salmon (D-S) PLUS staging. Compared with MR imaging, the results were as follows: PET and CT exhibited negative results, suggesting mild DI; one of them was positive, suggesting moderate DI; and two were positive, suggesting severe DI.
\end{abstract}

Correspondence to: Dr Jinhua Zhao, Department of Nuclear Medicine, Shanghai General Hospital of Nanjing Medical University, 100 Haining Road, Shanghai 200080, P.R. China

E-mail: zhaojinhua1963@126.com

Dr Jiqin Yang, Department of Nuclear Medicine, General Hospital of Ningxia Medical University, 804 Shengli Street, Yinchuan, Ningxia 750004, P.R. China

E-mail: qin-yj06@163.com

Key words: PET/CT, CT, multiple myeloma, bone marrow diffuse infiltration, focal lesion, false negative, Durie-Salmon PLUS staging system, Revised International Staging System
The results of concordance between two methods (new and routine) and MR imaging are indicated as follows: For the new method, McNemar test, $\mathrm{P}=0.513$ and Kappa=0.745; for the routine ${ }^{18} \mathrm{~F}-\mathrm{FDG} \mathrm{PET} / \mathrm{CT}$ method, McNemar test, $\mathrm{P}=0.03$ and Kappa=0.547. Re-performance of D-S PLUS staging presented the following results: New method, McNemar test, $\mathrm{P}=0.317$ and $\mathrm{Kappa}=0.93$; for the routine method, McNemar test, $\mathrm{P}=0.223$ and Kappa $=0.811$. These findings indicated that the CT component of PET/CT could improve the concordance with MRI results in the assessment of DI, and the same results were obtained when D-S PLUS staging was performed. The $\mathrm{CT}$ in PET/CT can enhance diagnostic accuracy in the assessment of DI by reducing the false negatives when compared with the routine ${ }^{18} \mathrm{~F}-\mathrm{FDG}$ method.

\section{Introduction}

Multiple myeloma (MM) is characterized by the infiltration and growth of malignant plasma cells in the bone marrow. When the signs of end-organ damage occur, systemic treatment should be initiated (1). Initially, Durie and Salmon determined that lytic bone lesions on radiographs, hemoglobin levels, serum calcium and monoclonal proteins were correlated with tumor mass and patient survival. The criteria of end-organ damage are based on the Durie-Salmon (D-S) staging system (2). Notably, this staging system has been widely used in recent decades. However, the primary limitation of X-ray modality is the requirement of a minimum of $30 \%$ trabecular bone involvement for lesion detectability (3). To assess bone disease with more sensitivity $(1,4)$, a newer D-S PLUS staging system including MR imaging and ${ }^{18} \mathrm{~F}-\mathrm{FDG}$ PET/CT was introduced by the International Myeloma Working Group (IMWG). The D-S PLUS staging primarily relies on two clinical factors: The number of focal lesions (FLs) and the diffuse infiltration (DI) level of bone marrow (Table I).

The main task for radiologists is to identify the number and extent of bone lesions via those sensitive imaging technologies. MR imaging of the spine or pelvis is used to evaluate the DI of MM, which is considered as the gold standard technique for assessing DI of the bone marrow, according 
Table I. D-S and D-S PLUS staging system.

\begin{tabular}{|c|c|c|}
\hline Stages & D-S staging system & D-S PLUS staging system \\
\hline I & $\begin{array}{l}\text { All of the following: Hemoglobin, }>10 \mathrm{~g} / \mathrm{dl} \text {; Albumin, } \\
>3.5 \mathrm{~g} / \mathrm{dl} \text {; Serum calcium, } \leq 12 \mathrm{mg} / \mathrm{dl} \text {; Low M-protein } \\
\text { concentration }(\mathrm{IgG},<5 \mathrm{~g} / \mathrm{d} ; \mathrm{IgA},<3 \mathrm{~g} / \mathrm{d} \text {; and Bence } \\
\text { Jones protein, }<4 \mathrm{~g} / 24 \mathrm{~h}) \text {; and X-ray, normal bone } \\
\text { structure or solitary bone plasmocytoma only. }\end{array}$ & $\begin{array}{l}\text { A: Normal skeletal survey or sing } \\
B^{\text {a. }} 0-4 \text { FLs or mild DI. }\end{array}$ \\
\hline II & Neither stage I nor stage III. & $\mathrm{A} / \mathrm{B}^{\mathrm{a}}$ : 5-20 FLs or moderate DI. \\
\hline III & $\begin{array}{l}\text { One or more of the following: Hemoglobin, }<8.5 \mathrm{~g} / \mathrm{dl} \text {; } \\
\text { Serum calcium, }<12 \mathrm{mg} / \mathrm{dl} \text {; extensive lytic bone lesions; } \\
\text { and high M-protein concentration ( } \operatorname{IgG}>7 \mathrm{~g} / \mathrm{dl} \text {, } \\
\mathrm{IgA}>5 \mathrm{~g} / \mathrm{dl} \text { or Bence Jones protein }>12 \mathrm{~g} / 24 \mathrm{~h} \text { ). }\end{array}$ & $\mathrm{A} / \mathrm{B}^{\mathrm{a}}:>20$ FLs or severe DI. \\
\hline
\end{tabular}

The Durie-Salmon PLUS staging system is subclassified A or B. $\mathrm{B}^{\mathrm{a}}$, a creatinine level $>2 \mathrm{mg} / \mathrm{dl}$ and/or EMD on PET/CT or MR imaging, as described in a previous study (6). D-S PLUS staging system, Durie-Salmon PLUS staging system; FL, focal lesions; DI, diffuse infiltration.

to a number of systematic reviews with large samples $(5,6)$. ${ }^{18} \mathrm{~F}-\mathrm{FDG}$ PET/CT is applied for FLs assessment more often than routine MR imaging, because of a larger field of view (FOV) and more availability than whole-body MR imaging. DI assessment (via MR imaging of the spine or pelvis) and FL assessment (via PET/CT) are combined to perform D-S PLUS staging. For simplified procedure or patients who have MR imaging contraindications, PET/CT alone is also used to perform one-stop D-S PLUS staging, that is, both FLs and DI can be assessed by PET/CT.

${ }^{18}$ F-FDG PET/CT has been considered a valuable tool for the evaluation of newly diagnosed and relapsed MM by the IMWG, the National Comprehensive Cancer Network of MM and the British Society for Haematology $(7,8)$. However, to the best of our knowledge, there is no available standard for the interpretation of PET/CT alone in D-S PLUS staging. To further promote the application of PET/CT, a group of nuclear medicine physicians and hematologists are attempting to formulate guidelines or criteria for imaging interpretation (9), in which only the levels of FDG uptake were adopted for DI assessment, while the CT component of PET/CT is not included. This may be unsuitable for patients with low or no uptake of ${ }^{18}$ F-FDG in lesions but with osteolytic destruction that can be manifested on CT imaging. In this study, we investigated a method for DI assessment that focused on the CT component of PET/CT for diagnostic purposes. In addition, the D-S PLUS staging system was compared with the Revised International Staging System (R-ISS) in the assessment of disease burden and prognosis at initial staging, the later relying primarily on laboratory parameters (10).

\section{Patients and methods}

Patients. This retrospective study was performed following approval by the Ethics Comittee of Shanghai General Hospital (Shanghai, China). The Ethics board waived informed patient consent. Patients were diagnosed with MM on the basis of the criteria defined by IMWG (11).

A total of 28 patients with untreated newly diagnosed MM between 2012 and 2017 were included in the present study. All patients underwent whole-body ${ }^{18} \mathrm{~F}-\mathrm{FDG}$ PET and vertebral column or pelvic MR imaging within a period of a week. The following staging and diagnostic data were simultaneously available for all patients: Whole blood count, routine biochemistry, serum $\beta 2$-microglobulin levels, C-reactive protein, serum and urinary immunofixation, complete immunoglobulin and serum-free light chain concentrations, $24 \mathrm{~h}$ proteinuria, bone marrow biopsy and chromosomal abnormalities detected by interphase fluorescent in situ hybridization (iFISH). The follow-up data of 4 patients could not be collected. The 24 remaining patients containing 6 women and 18 men with a mean age of 61 years (range from 24-77 years) were treated with therapeutic regimens including immunomodulatory agents or proteasome inhibitors. The treatment was subsequently followed by autologous bone marrow transplantation in 5 patients. The median follow-up time was 17.4 months (range from 1 to 42 months). Patients were followed up from the date of diagnosis up to May 2018 or when death occurred. Additionally, progression-free survival (PFS) was considered the endpoint. Image acquisition of PET/CT and MR imaging and image interpretation criterion are provided in Data $\mathrm{S} 1$.

Statistical analysis. The McNemar and Kappa tests were performed to evaluate the differences and concordance among the results, respectively. The degree of agreement by Kappa was interpreted as follows: Kappa $\geq 0.75$, substantial agreement; 0.75> Kappa $\geq 0.4$, moderate agreement; and Kappa $<0.4$, slight or fair agreement. Data were analyzed using SPSS 17.0 (SPSS, Inc.). $\mathrm{P}<0.05$ was considered to indicate a statistically significant difference. The PFS curves of D-S PLUS staging and R-ISS staging were estimated using the Kaplan-Meier method and compared using the log-rank test.

\section{Results}

The new method that considered the CT component of PET/CT for DI assessment, was classified into three levels according to the MR imaging results (Table II). The three levels of MRI are shown in Figs. S1-S3, respectively. The 

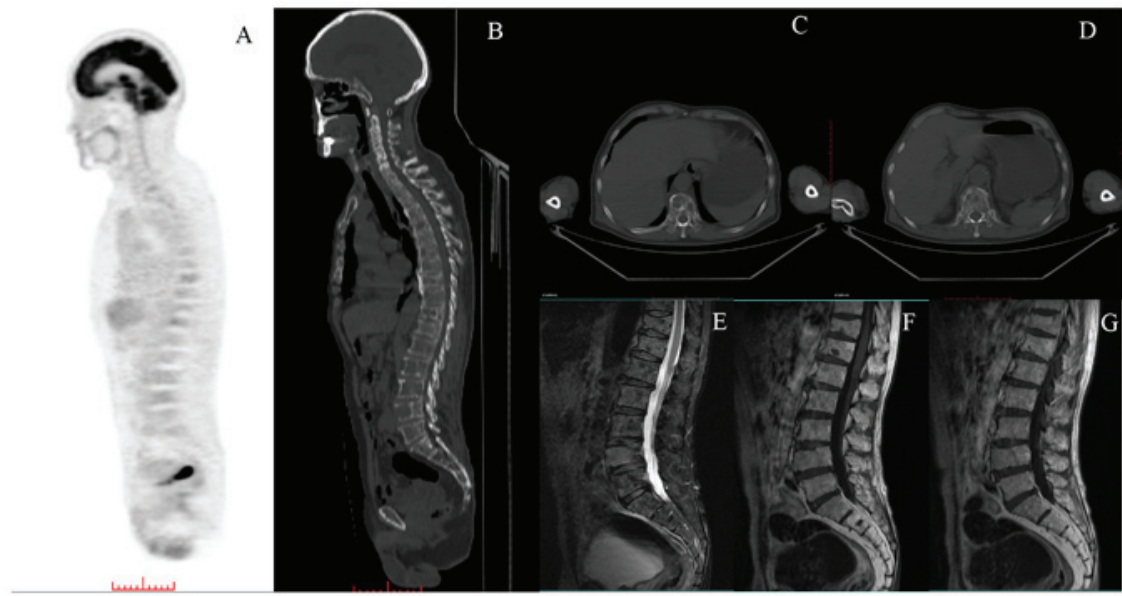

Figure 1. A 76-year-old male. (A) The ${ }^{18}$ F-FDG uptake of BM was no higher than that of liver, with the exception of the wedge-shaped T12. A FL on T9 shows mild FDG uptake ( $\leq$ liver uptake $+10 \%$ ). One unit on the scale bar represents $1 \mathrm{~cm}$, and the length of the scale in the figure represents the actual length of $10 \mathrm{~cm}$. (B) Sagittal CT image of whole-body demonstrated osteolytic type DI, which primarily manifested as diffuse circular bone destruction area. (C) Axial CT images of T10 and (D) T11, showed the bone destruction area with clear boundary, no hardening edge periosteal reaction and periosteal osteogenesis. (E) T2-TIRM, (F) T1WI and (G) T1WI+Contrast revealed heterogeneous diffuse bone marrow infiltration with T4 paramedullary disease. Both CT and MR imaging revealed positive DI of bone marrow, while the uptake of ${ }^{18} \mathrm{~F}$-FDG was negative. DI, diffuse infiltration.

Table II. Optimal combination of CT and PET component for DI assessment.

\begin{tabular}{lll}
\hline PET & CT & DI-PET/CT (stage) \\
\hline 0 & 0 & Mild DI \\
0 & 1 & Moderate DI \\
1 & 0 & Moderate DI \\
1 & 1 & Severe DI \\
\hline
\end{tabular}

0 , negative; 1 , positive; DI, diffuse infiltration.

P-value of the McNemar test between the new method and the MR imaging results was $\mathrm{P}=0.513$, suggesting no significant difference between the new method and MR imaging for DI assessment. The Kappa value was 0.745 , indicating moderate agreement (a Kappa value $\geq 0.75$ means substantial agreement). The routine method PET/CT, in which only the results of PET component were analyzed, was compared with MR imaging for the concordance of DI assessment. The P-value for the McNemar test was 0.03, showing a significant difference between the two methods, while the Kappa value was 0.547 indicating moderate agreement. This might be caused by using different methods to analyze the data. The Kappa value could be used as a reference for this test.

The new method and routine method of performing PET/CT for DI assessment were applied in the D-S PLUS staging system for comparison. MR imaging with the D-S PLUS staging was considered the control group for DI assessment. There was no significant difference between the new method and the control group ( $\mathrm{McNemar}$ test, $\mathrm{P}=0.317$ ). The Kappa value was 0.930 , suggesting substantial agreement. In addition, no significant difference was found between the routine method and the control group (McNemar test, $\mathrm{P}=0.223$ ). The Kappa value was 0.811 , suggesting substantial agreement. Notably, the Kappa value was lower than that of the new method (Kappa=0.93).

Thus, the results of new method PET/CT including the CT component for the diagnostic purpose of DI assessment were more consistent with these of MR imaging when compared with the routine method PET/CT, which consisted of only the levels of ${ }^{18} \mathrm{~F}-\mathrm{FDG}$ uptake (Kappa, 0.745 vs. 0.547). The same statistical outcomes could be obtained when the methods were considered for D-S PLUS staging (Kappa, 0.930 vs. 0.811). And CT manifested 3 modes in DI of MM in our study, Osteolytic type, osteoporosis type and insect-corroded osteolytic type, shown on Figs. 1-3 respectively, which will be discussed in detail later.

The PFS curves showed that both current D-S PLUS staging with MR imaging for DI assessment and R-ISS staging had good prognostic value (D-S PLUS staging: I vs. II, $\mathrm{P}=0.016$; I vs. III, $\mathrm{P}=0.001$; and II vs. III, $\mathrm{P}=0.0003$; R-ISS staging: I vs. II, $\mathrm{P}=0.036$; I vs. III, $\mathrm{P}=0.001$; and II vs. III, $\mathrm{P}=0.0002$ ), which were shown in Figs. 4 and 5. The P-value of McNemar test for D-S PLUS stage system and R-ISS stage system was 0.223 , indicating no significant difference between them. Furthermore, a Kappa value of 0.6789 (0.4 Kappa <0.75) showed moderate agreement.

\section{Discussion}

MR imaging is considered as the gold standard for DI assessment. Due to the infiltration of the bone marrow via displacement of fat cells by neoplastic cells, the lesions can be visualized as areas of low signal intensity on T1-weighted spin-echo images and high signal intensity on T2 fat-suppressed images, when the tumor cells accumulate to a certain extent.

The levels of ${ }^{18}$ F-FDG uptake can reflect the activity of neoplastic cells. In a consensus statement by the IMWG in 2017, 12 studies containing 859 patients reported the sensitivity and specificity of ${ }^{18} \mathrm{~F}$-FDG PET in detecting bone damage caused by neoplastic cells (80-100\%). For CT imaging, a certain amount of time must pass before the osteoclastic activity of 

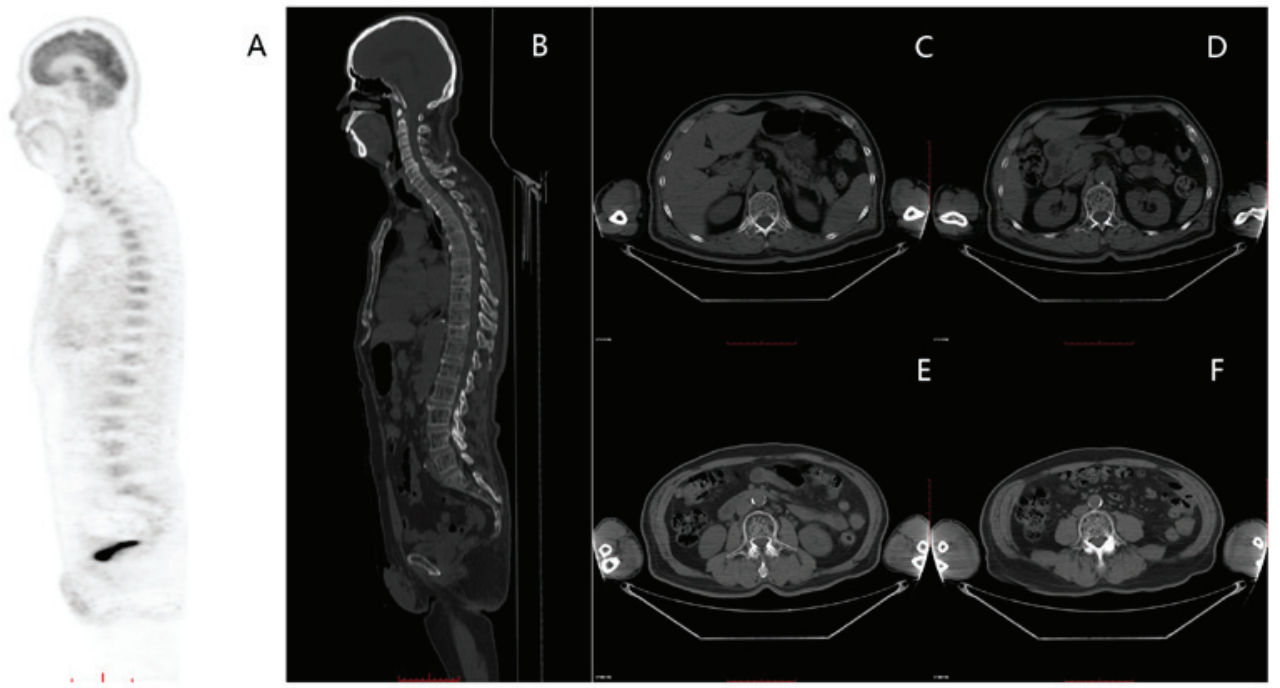

Figure 2. A 64-year-old male. (A) The ${ }^{18} \mathrm{~F}-\mathrm{FDG}$ uptake of BM was higher than that of liver, (B) sagittal CT image of whole-body demonstrated osteoporosis type DI, the bone density was generally reduced, (C) axial CT images of T12, (D) L1, (E) L3 and (F) L4 showed that the bone ridge was sparse, the residual bone trabecular structure was irregular and thickened, and there were no sign of obvious bone damage. Biopsy confirmed MM invasion. MM, Multiple myeloma.

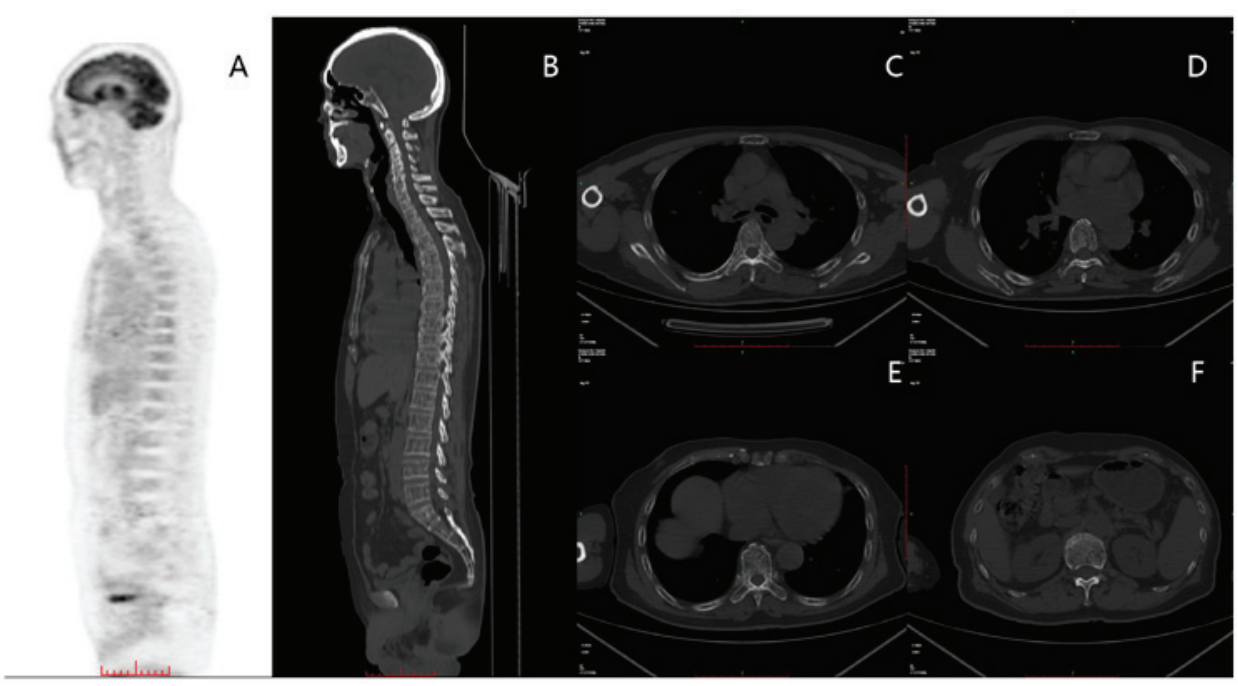

Figure 3. A 76-year-old male. (A) The ${ }^{18} \mathrm{~F}$-FDG uptake of BM was higher than that of liver, (B) sagittal CT image of whole-body demonstrated insect-corroded osteolytic type DI, which showed 'Moth-eaten' bone invasion, (C) axial CT images of T5, (D) T7, (E) T10, (F) T12, and the vertebral appendages, ribs, sternum, scapula within scanning rang showed the permeative 'punched-out' lacunae on bone with clear boundaries and no hardening. Biopsy confirmed MM invasion.

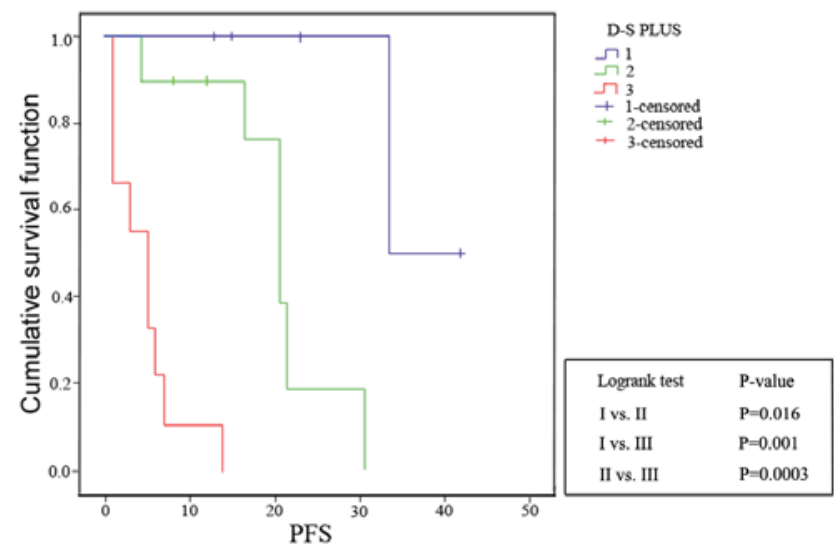

Figure 4. The blue color curve represents stage I, green color curve represents stage II, red color curve represents stage III. PFS, progression-free survival; D-S, Durie-Salmon.

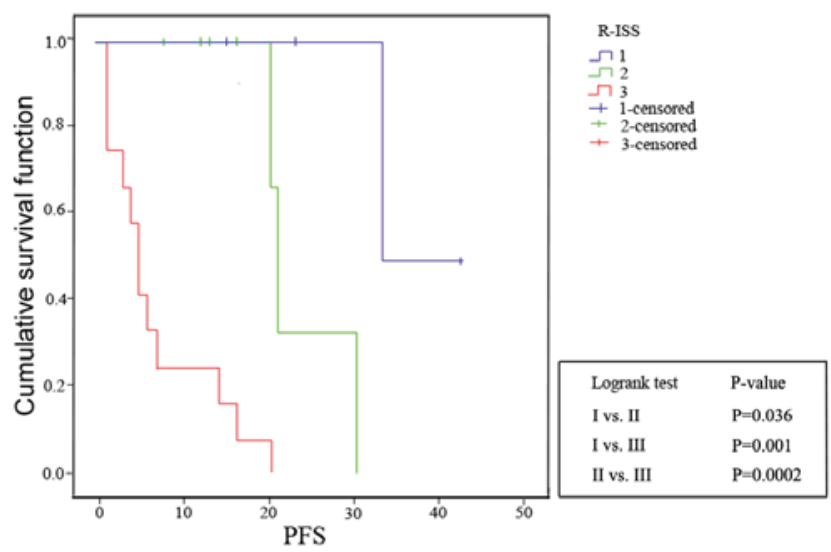

Figure 5. The blue color curve represents stage I, green color curve represents stage II, red color curve represents stage III. R-ISS, Revised International Staging System staging system. 
myeloma cells leads to the destruction of trabecular or cortical bone (12). Thus, in the majority of PET/CT studies concerning DI of bone marrow, only the PET component of PET/CT is considered to be able to identify the progression of DI. While the CT component of PET/CT has not been widely considered for diagnostic purposes in the assessment of DI, and only used for primary level attenuation correction and focal localization in PET imaging. In a systematic review concerning diagnostic performance of whole-body MR imaging and ${ }^{18} \mathrm{~F}-\mathrm{FDG}$ PET/CT in patients with myeloma, only one study considered the CT component of PET/CT for reference standard of assessing lesions, and the others focused on the PET component only (13). However, the PET component does not always hold true. Taking a case from our study for example, all MR imaging, CT results and pathological tests revealed positive DI of bone marrow, however, the uptake of ${ }^{18} \mathrm{~F}-\mathrm{FDG}$ was negative. These results indicated that the ${ }^{18} \mathrm{~F}-\mathrm{FDG}$ uptake of myeloma cells might be suppressed, leading to an inferior DI assessment by PET/CT when compared with MR imaging.

The CT component of PET/CT can aid the assessment of DI cases with no or low ${ }^{18} \mathrm{~F}$-FDG uptake, although it may be still negative when the early MM does not present bone destruction. In our study, compared with the routine method without the CT component of PET/CT, the new method that considered the CT component of PET/CT has a better consistency with MR imaging (Kappa, 0.745 vs. 0.547). The same results could be obtained when performed D-S PLUS staging.

According to the European Myeloma Network Guidelines, the grading levels of evidence of $\mathrm{CT}$ in evaluating bone destruction lesions is $1 \mathrm{~A}$ compared with that of the traditional $\mathrm{X}$-ray (14). However, CT evaluation of bone marrow invasion also requires careful identification. In the analysis of cases in this study, we found that diffuse bone marrow invasion and positive manifestations of MM were mainly divided into three modes on CT, Osteolytic type, osteoporosis type and insect-corroded osteolytic type, and illustrated in the Results. The first type requires identification via osteoclastic metastatic tumors and is mainly manifested as diffuse circular bone destruction area with clear boundary, no hardening edge and periosteal osteogenesis; The second needs to be distinguished from osteoporosis caused by senile non-myelogenous, long-term alcoholism and hormone therapy, and is mainly observed with reduced bone density, sparse bone ridges and irregular thickening of residual bone trabeculae without obvious bone damage; The last identified via osteolytic bone damage with clear boundaries and no hardening, is diffuse and punctured. All the above types can be combined with pathological fractures and should be identified via fractures caused by osteoporosis.

It is well known that hyperglycemia and the recent administration of high-dose steroids can lead to a transient metabolic suppression. Besides that, there may be some other reasons associated with the intrinsic features of myeloma cells. Several studies have shown that myeloma cells might not be ${ }^{18}$ F-FDG-avid (15-17). In the study by Rasche et al, 227 newly diagnosed MM patients were prospectively and simultaneously studied at baseline with diffusion weighted imaging (DWI) whole-body MR imaging and ${ }^{18} \mathrm{~F}-\mathrm{FDG}$ PET, along with iFISH and gene expression profiling (GEP) (18). The researchers found that $11 \%$ of those patients were PET false-negative/DWI MR imaging-positive, and none of these patients belonged to the GEP-defined hyperdiploid or proliferative molecular subgroup. Most importantly, in those 21 differentially expressed genes between the PET false-negative/DWI MR imaging-positive group and PET-positive/DWI MR imaging-positive group, the top gene was the one coding for hexokinase-2, and its expression was significantly lower in the PET false-negative group. Furthermore, deregulation of 5 other genes involved in metabolism was also observed (18). The reason for the decline of related genes regulation remains to be further studied. According to the reverse Warburg effect, aerobic glycolysis takes place in tumor-associated fibroblasts but not in cancer cells. Therefore, we can speculate that the level of ${ }^{18} \mathrm{~F}-\mathrm{FDG}$ uptake of DI may be associated with the oxygen content in the microenvironment of bone marrow and vary with the different processes of MM. However, such hypotheses have not been explored in ${ }^{18} \mathrm{~F}-\mathrm{FDG}$ studies.

In addition to false negatives, false positives of ${ }^{18} \mathrm{~F}-\mathrm{FDG}$ uptake can occasionally be found in routine clinical practice. Most of false positives are associated with increased red bone marrow conversion due to anemia, and the false positives caused by bone marrow conversion are difficult for antidiastole with DI of tumor cells in either PET/CT or MR imaging. Prospective trials that extensively apply novel techniques must be performed. At present, researchers have been investigating new PET/CT tracers that target different metabolic pathways or receptors expressed by MM cells to improve the sensitivity and specificity of imaging modalities in the D-S PLUS stage system. Preliminary results have been recorded $(19,20)$.

In the present study, both the D-S PLUS staging systems mainly relying on imaging findings and R-ISS staging systems basing on clinical laboratory data have good prognostic value for PFS. However, the former is considered more intuitive and stable, particularly useful for monitoring disease status and comparing treatment response in non-secretory patients $(21,22)$. Due to the limitation of the sample size, we did not analyze the rate of PET/CT false negatives in detail because the small numbers might lead to statistical bias. In addition, the diffuse bone marrow invasion performance by $\mathrm{CT}$ might not be exhaustive in previously discussion. To provide more reliable evidence for clinical practice, larger studies are required.

In conclusion, the inclusion of CT findings from PET/CT for diagnostic purposes can further improve the ability to assess DI by reducing the false negatives when compared with the routine PET/CT method, especially in those cases with no or low avidity to ${ }^{18} \mathrm{~F}$-FDG. Notably, the CT findings can be false negatives when the bone trabeculae has not been destroyed in early MM. Implementing this method may be helpful for clinicians when considering PET/CT for one-stop D-S PLUS staging in cases where patients have contraindications for MR imaging or if whole-body MR imaging is not available.

\section{Acknowledgements}

Not applicable.

\section{Funding}

This study was supported by the Shanghai Science and Technology Commission Research Project (grant 
no. 17411953200); the Shanghai Shenkang Hospital Development Center Clinical Auxiliary Departments Capacity Building Project (grant no. SHDC22015032).

\section{Availability of data and materials}

The datasets used and analyzed during the present study are available from the corresponding author on reasonable request.

\section{Authors' contributions}

JZ and JY conceived and designed the present study. TW and $\mathrm{XP}$ performed the experiments and wrote the manuscript. TW, JY and WQ acquired the data. XP and YX analyzed and interpreted the data. TW, WQ and YX performed statistical analysis. JZ revised the manuscript for important intellectual content. All authors read and approved the final manuscript.

\section{Ethics approval and consent to participate}

This retrospective study was performed following approval by the Ethics Comittee of Shanghai General Hospital (Shanghai, China). The Ethics board waived informed patient consent. (approval no. 2018KY167).

\section{Patient consent for publication}

Not applicable.

\section{Competing interests}

The authors declare that they have no competing interests.

\section{References}

1. Durie BG, Kyle RA,Belch A, Bensinger W, Blade J, Boccadoro M, Child JA, Comenzo R, Djulbegovic B, Fantl D, et al: Myeloma management guidelines: A consensus report from the scientific advisors of the International Myeloma Foundation. Hematol J 4 379-398, 2003.

2. Dimopoulos M, Kyle R, Fermand JP, Rajkumar SV, San Miguel J, Chanan-Khan A, Ludwig H, Joshua D, Mehta J, Gertz M, et al: Consensus recommendations for standard investigative workup: Report of the International Myeloma Workshop Consensus Panel 3. Blood 117: 4701-4705, 2011.

3. Durie BG and Salmon SE: A clinical staging system for multiple myeloma. Correlation of measured myeloma cell mass with presenting clinical features, response to treatment, and survival. Cancer 36: 842-854, 1975.

4. International Myeloma Working Group: Criteria for the classification of monoclonal gammopathies, multiple myeloma and related disorders: A report of the International Myeloma Working Group. Br J Haematol 121: 749-757, 2003.

5. Mesguich C, Fardanesh R, Tanenbaum L, Chari A, Jagannath S and Kostakoglu L: State of the art imaging of multiple myeloma: Comparative review of FDG PET/CT imaging in various clinical settings. Eur J Radio 83: 2203-2223, 2014.

6. van Lammeren-Venema D, Regelink JC, Riphagen II, Zweegman S, Hoekstra OS and Zijlstra JM: ${ }^{18}$ F-Fluoro-deoxyglucose positron emission tomography in assessment of myeloma-related bone disease: A systematic review. Cancer 118: 1971-1981, 2012.

7. Cavo M, Terpos E, Nanni C, Moreau P, Lentzsch S, Zweegman S, Hillengass J, Engelhardt M, Usmani SZ, Vesole DH, et al: Role of ${ }^{18} \mathrm{~F}$-FDG PET/CT in the diagnosis and management of multiple myeloma and other plasma cell disorders: A consensus statement by the International Myeloma Working Group. Lancet Oncol 18: e206-e217, 2017.
8. Chantry A, Kazmi M, Barrington S, Goh V, Mulholland N, Streetly M, Lai M and Pratt G; British Society for Haematology Guidelines: Guidelines for the use of imaging in the management of patients with myeloma. Br J Haematol 178: 380-393, 2017.

9. Nanni C, Versari A, Chauvie S, Bertone E, Bianchi A, Rensi M, Bellò M, Gallamini A, Patriarca F, Gay F, et al: Interpretation criteria for FDG PET/CT in multiple myeloma (IMPeTUs): Final results. IMPeTUs (Italian myeloma criteria for PET USe). Eur J Nucl Med Mol Imaging 45: 712-719, 2018.

10. Palumbo A, Avet-Loiseau H, Oliva S, Lokhorst HM, Goldschmidt H, Rosinol L, Richardson P, Caltagirone S, Lahuerta JJ, Facon T, et al: Revised international staging system for multiple myeloma: A report from international myeloma working group. J Clin Oncol 33: 2863-2869, 2015.

11. Rajkumar SV, Dimopoulos MA, Palumbo A, Blade J, Merlini G, Mateos MV, Kumar S, Hillengass J, Kastritis E, Richardson P, et al: International Myeloma Working Group updated criteria for the diagnosis of multiple myeloma. Lancet Oncol 15: e538-e548, 2014

12. Baur-Melnyk A, Buhmann S, Becker C, Schoenberg SO, Lang N, Bartl R and Reiser MF: Whole-body MRI versus whole-body MDCT for staging of multiple myeloma. AJR Am J Roentgenol 190: 1097-1104, 2008.

13. Gariani J, Westerland O, Natas S, Verma H, Cook G and Goh V: Comparison of whole body magnetic resonance imaging (WBMR imaging) to whole body computed tomography (WBCT) or ${ }^{18} \mathrm{~F}$-fluorodeoxyglucose positron emission tomography/CT $\left({ }^{18} \mathrm{~F}-\mathrm{FDG}\right.$ PET/CT) in patients with myeloma: Systematic review of diagnostic performance. Crit Rev Oncol Hematol 124: 66-72, 2018.

14. Terpos E, Kleber M, Engelhardt M, Zweegman S, Gay F, Kastritis E, van de Donk NW, Bruno B, Sezer O, Broijl A, et al: European Myeloma Network guidelines for the management of multiple myeloma-related complications. Haematologica 100: 1254-1266, 2015

15. Zamagni E, Patriarca F, Nanni C, Zannetti B, Englaro E, Pezzi A, Tacchetti P, Buttignol S, Perrone G, Brioli A, et al: Prognostic relevance of 18 -F FDG PET/CT in newly diagnosed multiple myeloma patients treated with up-front autologous transplantation. Blood 118: 5989-5995, 2011.

16. Bartel TB, Haessler J, Brown TL, Shaughnessy JD Jr, van Rhee F, Anaissie E, Alpe T, Angtuaco E, Walker R, Epstein J, et al: F18-fluorodeoxyglucose positron emission tomography in the context of other imaging techniques and prognostic factors in multiple myeloma. Blood 114: 2068-2076, 2009.

17. Nanni C, Zamagni E, Celli M, Caroli P, Ambrosini V, Tacchetti P, Brioli A, Zannetti B, Pezzi A, Pantani L, et al: The value of ${ }^{18} \mathrm{~F}$-FDG PET/CT after autologous stem cell transplantation (ASCT) in patients affected by multiple myeloma (MM): Experience with 77 patients. Clin Nucl Med 38: e74-e79, 2013.

18. Rasche L, Angtuaco E, McDonald JE, Buros A, Stein C, Pawlyn C, Thanendrarajan S, Schinke C, Samant R, Yaccoby S, et al: Low expression of hexokinase-2 is associated with false-negative FDG-positron emission tomography in multiple myeloma. Blood 130: 30-34, 2017.

19. Herrmann K, Schottelius M, Lapa C, Osl T, Poschenrieder A, Hänscheid H, Lückerath K, Schreder M, Bluemel C, Knott M, et al: First-in-human experience of CXCR4-Directed endoradiotherapy with ${ }^{177} \mathrm{Lu}$ - and ${ }^{90}$ Y-Labeled pentixather in advanced-stage multiple myeloma with extensive intra- and extramedullary disease. J Nucl Med 57: 248-251, 2016.

20. Okasaki M, Kubota K, Minamimoto R, Miyata Y, Morooka M, Ito K, Ishiwata K, Toyohara J, Inoue T, Hirai R, et al: Comparison of (11)C-4'-thiothymidine, (11)C-methionine, and (18)F-FDG $\mathrm{PET} / \mathrm{CT}$ for the detection of active lesions of multiple myeloma. Ann Nucl Med 29: 224-232, 2015.

21. Lonial S and Kaufman JL: Non-secretory myeloma: A clinician's guide. Oncology (Williston Park) 27: 924-928, 930, 2013.

22. Dammacco F, Rubini G, Ferrari C, Vacca A and Racanelli V: ${ }^{18} \mathrm{~F}-\mathrm{FDG}$ PET/CT: A review of diagnostic and prognostic features in multiple myeloma and related disorders. Clin Exp Med 15: 1-18, 2015. 